\title{
Técnicas para el manejo del tejido gingival en prótesis fija. Una revisión sistemática
}

\section{Methods used in order to manage the gingival tissue in fixed prostheses. A systematic review}

Cruz González AC*, Díaz Caballero A**, Méndez Silva JE***

\section{RESUMEN}

Antecedentes: Durante los tratamientos protésicos fijos, el proceso de impresión de dientes pilares puede verse afectado por la encía marginal, la hemorragia y los fluidos gingivales. El propósito de la retracción gingival es efectuar un desplazamiento temporal del margen gingival y control de los fluidos, para exponer adecuadamente la línea de terminación. Métodos de acción mecánica, mecánica-química y del campo de la cirugía son propuestos para este fin.

Objetivo: Efectuar una revisión de literatura crítica sobre los distintos métodos de retracción gingival, al considerar la posibilidad de lesiones en los tejidos blandos y la efectividad de estos.

Metodología: Se desarrolló una búsqueda de literatura en las bases de datos sciverse (science direct), Ovid (MEDLINE), Pubmed, Dentistry \& Oral Sciences Source (EBSCO-Host) y Google scholar, desde 1970 hasta diciembre de 2011. Con palabras claves como: Gingival retraction, gingival retraction methods, retraction cords, retraction agents, electrosurgery, tissue displacement. Una vez obtenida una muestra, se efectuó la confrontación con la literatura por medio de una discusión.

Resultados y discusión: El sondeo de artículos bajo ciertos criterios permitió la selección final de una muestra de 8 artículos. Las comparaciones sobre resultados clínicos e interacción con los tejidos son frecuentes, y los resultados no son homogéneos, por lo que distintos puntos de vista y tratamientos son sugeridos.

Palabras clave: Encía, hemostasia, técnica de impresión dental, fluido del surco gingival, electrocirugía, terapia por láser. (Decs Bireme).

\section{SUMMARY}

Background: During the fixed prosthetic treatments, the printing process abutment teeth can be affected by the marginal gingiva, bleeding and gingival fluids. The purpose of gingival retraction is to get a temporary displacement of the gingival margin and control of fluids, from properly expose the finish line. Methods of mechanical action, chemical and mechanical-surgery field are proposed for this purpose.

Objective: We conducted a critical literature review on the different methods of gingival retraction, when considering the possibility of damage soft tissue and the effectiveness to them. Methods: We developed a literature search in the databases sciverse (science direct), Ovid (MEDLINE), PubMed, Dentistry \& Oral Sciences Source (EBSCO-Host) and Google Scholar, since 1970 to December 2011. Using keywords like: Gingival

* Odontólogo. Universidad de Cartagena. Consultor.

** Odontólogo. Universidad de Cartagena. Periodoncista Universidad Javeriana. Maestría en Educación Universidad del Norte. Estudiante de doctorado en Ciencias Biomédicas Universidad de Cartagena. Profesor titular Universidad de Cartagena. Director Grupo de Investigaciones GITOUC.

*** Odontólogo. Universidad de Cartagena. Rehabilitador oral Universidad de Buenos Aires. Implantólogo oral Universidad Católica Argentina. Especialista en pedagogía para el desarrollo del aprendizaje autónomo Universidad Nacional a distancias. Profesor Asistente Universidad de Cartagena. 
retraction, gingival retraction Methods, retraction cords, retraction agents, electrosurgery, tissue displacement. After obtaining a sample, we was compared it with the literature through a discussion.

Results and discussion: The survey of articles under certain criteria it allowed the final selection of a sample of 6articles. Comparisons of clinical outcomes and interaction with tissue are frequent, and there are not results homogeneous, so that different positions and treatments are suggested.

Key words: Gingiva, hemostasis, dental impression technique, gingival crevicular fluid, electrosurgery, laser therapy. (MeSH DATABASE).

Fecha de recepción: 27 de diciembre de 2011.

Aceptado para publicación: 20 de enero de 2012.

Cruz González AC, Díaz Caballero A, Méndez Silva JE. Técnicas para el manejo del tejido gingival en prótesis fija. Una revisión sistemática. Av. Odontoestomatol 2013; 29 (4): 191-199.

\section{INTRODUCCIÓN}

En prótesis fija un ajuste preciso sobre el diente preparado es primordial para el éxito de la restauración (1). Durante la toma de impresión para restauraciones con márgenes subgingivales se busca obtener una reproducción inequívoca de la línea terminal y el surco gingival, con esto se pretende evitar futuras discrepancias entre el margen de la restauración y la preparación dental (2). Pero, la encía marginal, la hemorragia y los fluidos gingivales pueden representar un obstáculo para el acceso del material de impresión al interior del surco (3); con el fin de lograr un desplazamiento efectivo de dicho margen libre y el control de los fluidos, se reportan múltiples métodos de retracción gingival mecánicos, mecánicoquímicos y quirúrgicos. Las técnicas mecánicas incluyen el uso de bandas de cobre para el control de los fluidos y tejidos blandos alrededor del diente preparado (4). Otra alternativa propuesta son coronas provisionales en resina acrílica, que actúan mecánicamente como las bandas de cobre (5). El método mecánico-químico implica el desplazamiento del tejido gingival físicamente por un cordón, el cual es previamente impregnado por sustancias químicas para inducir la vasoconstricción y astringencia en los tejidos (6-8). En la retracción quirúrgica se pueden emplear fresas de diamante para dar una mejor conformación a la pared interna de la encía marginal, al tiempo que se prepara la línea terminal, método denominado curetaje gingival rotatorio (9). Otras alternativas son la electrocirugía y cirugía láser usadas para ampliar el espacio de surco gingival y expo- ner la línea terminal $(4,5,9)$. Estas técnicas quirúrgicas requieren de una encía saludable con un ancho considerable y un alto grado de queratinización para evitar futuras pérdidas en los niveles de inserción posteriores a la cirugía (10). Sin embargo, aun cuando las técnicas mencionadas pueden efectuar un adecuado desplazamiento, su inocuidad al tejido no es total, por lo que la posibilidad de lesiones durante el procedimiento debe ser considerada $(4,10)$.

\section{MATERIALES Y MÉTODOS}

Se desarrolló una revisión de literatura sistemática soportada en las bases de datos electrónicas: sciverse (science direct), Ovid (MEDLINE), Pubmed, Dentistry $\varepsilon$ Oral Sciences Source (EBSCO-Host) y Google scholar; para un periodo de 1970 a diciembre del 2011. La búsqueda se llevó a cabo mediante el uso de palabras claves como: Gingival retraction, gingival retraction methods, retraction cords, retraction agents, electrosurgery, tissue displacement. La selección se limitó a artículos completos en ingles, registrados en Pubmed, donde se comparan los distintos métodos de retracción y sus efectos, en condiciones in vivo, en pacientes humanos, además que se detallara la metodología seguida. De la totalidad de artículos obtenidos (Tabla 1) una filtración de la búsqueda determinó 65 resultados inicialmente, 54 textos completos y 11 resúmenes. Luego, una depuración final del contenido condujo a la selección final de 6 artículos, para ser analizados y confrontados por medio de una discusión. 


\begin{tabular}{|c|c|c|c|c|c|c|}
\hline \multicolumn{7}{|c|}{ TABLA 1} \\
\hline & Science Direct & Ovid & Pubmed & EBSCO-Host & Google scholar & Total \\
\hline 1. Gingival retraction & 280 & 256 & 278 & 2 & 113 & 929 \\
\hline 2. Gingival retraction methods & 20 & 0 & 103 & 0 & 4 & 127 \\
\hline 3. Retraction cords & 287 & 11 & 40 & 0 & 29 & 367 \\
\hline 4. Retraction agents & 24 & 13 & 11 & 1 & 16 & 65 \\
\hline 5. Electrosurgery & 3.665 & 4.225 & 3.521 & 388 & 924 & 12.723 \\
\hline 6. Tissue displacement & 1.206 & 355 & 193 & 90 & 71 & 1915 \\
\hline 1 y 2 AND & 20 & 229 & 30 & 2 & & 281 \\
\hline 1 y 3 AND & 132 & 36 & 43 & 0 & & 211 \\
\hline 1 y 4 AND & 17 & 9 & 31 & 0 & & 57 \\
\hline 1 y 5 AND & 44 & 13 & 21 & 0 & & 78 \\
\hline 1 y 6 AND & 28 & 4 & 8 & 0 & & 40 \\
\hline 2 y 3 AND & 10 & 0 & 14 & 0 & & 24 \\
\hline 2 y 4 AND & 2 & 1 & 14 & 0 & & 17 \\
\hline 2 y 5 AND & 8 & 2 & 11 & 1 & & 22 \\
\hline 2 y 6 AND & 4 & 1 & 6 & 0 & & 11 \\
\hline 3 y 4 AND & 297 & 2 & 16 & 9 & & 324 \\
\hline 3 y 5 AND & 40 & 6 & 4 & 0 & & 50 \\
\hline 3 y 6 AND & 27 & 2 & 2 & 0 & & 31 \\
\hline 4 y 5 AND & 7 & 0 & 1 & 0 & & 8 \\
\hline 4 y 6 AND & 11 & 1 & 2 & 0 & & 14 \\
\hline 5 y 6 AND & 17 & 3 & 4 & 0 & & 24 \\
\hline Total & 6.146 & 5.169 & 4.353 & 493 & 1.157 & 17.318 \\
\hline
\end{tabular}

\section{RESULTADOS Y DISCUSIÓN}

El objetivo principal de la retracción se basa en el desplazamiento reversible de los tejidos gingivales en dirección lateral, que asegure la entrada del material de impresión de baja viscosidad, permita el registro con precisión del área intracrevicular y permita un grosor adecuado de la impresión que disminuya el riesgo de desgarre al ser removida (11).
Para comprender mejor el movimiento que ocurre en la encía durante este procedimiento se deben revisar las fuerzas involucradas. La literatura reporta cuatro fuerzas resultantes de la interacción de la encía con los materiales de retracción e impresión: fuerza de retracción, recidiva, desplazamiento y colapso. La fuerza de retracción se describe como un movimiento hacia abajo y afuera del margen gingival libre, al desplazar la encía de los dientes preparados. La fuer- 
za recidivante es inherente del tejido gingival, se entiende como la tendencia de la encía a regresar a su posición original, es influenciada por la elasticidad gingival y la capacidad de recuperación de la encía adherida adyacente, comprimida durante la retracción. La fuerza de desplazamiento ocurre por el apoyo hacia abajo y consistencia del material de impresión directamente sobre el tejido gingival. El colapso tiene lugar durante la toma de impresión, al no existir un material de retracción en el interior del surco, el material de impresión contacta con el margen gingival libre y el tejido colapsa contra el diente preparado (12-14).

Inicialmente se emplearon métodos de acción exclusivamente mecánica como bandas de cobre (4), coronas provisionales en resina acrílica (5), polímeros biocompatibles (14) y sistemas de matriz con materiales de impresión elastoméricos para vencer dichas fuerzas (15). Pero se informa el uso ampliamente extendido de agentes químicos en asociación con hilos de diferentes diámetros, para lograr el desplazamiento de tejidos al tiempo que se controla la hemorragia y la absorción de fluidos, por vasoconstricción y astringencia (16-19). Estos hilos pueden emplearse de forma simple o por técnica doble, ambos por 8 a 10 minutos; con esta última se considera que al sumar un segundo hilo de mayor diámetro al primero en el surco, se pueden mejorar los resultados esperados (19). Por otro lado, varios son los agentes químicos reportados para este fin como la epinefrina, cloruro de aluminio, sulfato de aluminio, sulfato férrico y algunos miembros del grupo de las aminas simpaticomiméticas como tetrahidrozolina, oximetazolina, Clorhidrato de fenilefrina $(6-8,20)$.

En 1984, Weir y Williams (21) compararon la efectividad de tres tipos de hilo retractor del mismo calibre, con contenidos de 0,60 mg de epinefrina, 2,60 mg de sulfato de aluminio y uno sin impregnado, en 120 dientes de 58 pacientes evaluados para coronas completas. Los hilos se usaron de tres formas: secos, saturados con agua destilada y en una solución de cloruro de aluminio (Hemodent). La evaluación comprendió, la ocurrencia o no de sangrado al remover el hilo y el control o no de hemorragia en un minuto. A partir de esto no se determinó diferencia significativa entre los hilos con epinefrina y sulfato de aluminio, a su vez estos fueron más efectivos que el grupo de no impregnados. El control de la hemorragia del grupo con Hemodent fue más efectivo que el grupo de hilos secos o saturados de agua destilada. De esto concluyeron que se puede considerar eliminar el uso de un agente con riesgo de efecto sistémico por otros más inocuos, además Hemodent aproximadamente duplico el éxito de hemostasia de los otros grupos estudiados.

Un año después, Donovan et al (22), a través de un estudio y revisión literaria, determinan que, de los método de retracción, el más usado en una muestra de 495 dentistas fue el mecánico-químico con el hilo retractor con epinefrina, además concluyen que la epinefrina representa un potencial riesgo por sus efectos sistémicos, debido a la absorción local en función de la exposición del sistema circulatorio, e informan de la posibilidad de uso alternativo del sulfato de aluminio y cloruro de aluminio al $10 \%$ al disminuir el riesgo de efectos sistémicos.

En un estudio más reciente, Csillag et al 2007 (23), al evaluar tres grupos de hilos retractores saturados con epinefrina en concentraciones de $0,1 \%, 0,01 \%$, $0,001 \%$, y uno con solución salina como control, en incisivos centrales superiores izquierdos de $17 \mathrm{pa}-$ cientes humanos, determinó que después de remover los hilos con epinefrina con $0,1 \%, 0,01 \%$ los niveles de sangrado y fluido crevicular se mantuvieron bajos. Aunque informó de una elevación transitoria del fluido en el grupo de $0,01 \%$, que luego que se estableció al nivel del grupo del 0,1\%. En el grupo control reporto elevación del sangrado y fluido, también se informó la ausencia de efectos vasculares sistémicos. Por lo que concluyó que posiblemente con $0,01 \%$ de epinefrina en el hilo retractor puede detenerse la hiperemia y producción de fluido crevicular para la toma de impresión, sin afectar los niveles circulatorios sistémicos o causar isquemia prolongada.

Otros reportes de la literatura también informan del uso de epinefrina en concentraciones de 0,1 al $8 \%$, pero existen controversias sobre su uso debido a la posibilidad de efectos vasculares sistémicos, algunos sugieren el uso de otros agentes, ya mencionados, como el sulfato de aluminio al $25 \%$ y cloruro de aluminio de 10-25\% (24-26). Estos últimos se consideran como astringentes de acción similar, reportan 
una acción hemostática por medio de la precipitación de proteínas en el tejido y una vasoconstricción en menor grado que la epinefrina (12).

Una solución de sulfato férrico al 15,5\% en hilos de retracción, por 5 a 10 minutos en el surco, también puede lograr la hemostasia al permitir una estabilización del coagulo, manifestándose esto, con una coloración temporal entre amarillo-marrón y negro en la encía cercana al surco $(19,27)$. Pero se reporta que la reproducción final con materiales de impresión como el ponivinil siloxano puede ser afectada por el sulfato férrico, al interferir en s polimerización; por lo que se recomienda lavar muy bien el surco después de retirar el cordón retractor con este agente $(20,27)$. Otras posibles opciones incluyen sustancias usadas en descongestionantes nasales u oftálmicos, como el clorhidrato de tetrahidrozolina al $0,05 \%$, clorhidrato oximetazolina al $0,05 \%$ y clorhidrato de fenilefrina al 0,25\%, pertenecientes al grupo de las aminas simpaticomiméticas, capaces de producir un vasoconstricción local con mínimos efectos sistémicos (6). En el 2002, Kopac et al (28) evaluaron y compararon los posibles efectos de la tetrahidrozolina al $0,05 \%$ y el cloruro de aluminio $25 \%$, durante 10 minutos de uso, en cultivos de queratinocitos provenientes de la encía de ratas. Determinaron que el cloruro de aluminio fue significativamente más agresivo que la tetrahidrozolina, al reportar un daño celular mayor y extenso. Por lo que informan de la posibilidad de usar la tetrahidrozolina al 0,05\% en los tratamientos clínicos en prótesis fija. A pesar de esto se reporta la posibilidad de interferencia en la polimerización en materiales de impresión por parte de algunos de estos agentes al igual que con el cloruro de aluminio y sulfato férrico (8).

Una propuesta alternativa ante el método de cordones impregnados es el uso de una matriz inyectable con la consistencia de una pasta, a base de cloruro de aluminio y caolín, que busca realizar la retracción disminuyendo el trauma excesivo a los tejidos (10, 29). Los posibles efectos de este método mecánicoquímicos y su efectividad son comparados con el hilo retractor a través de múltiples investigaciones.

Acerca de esto, Al Hamad et al (30) en un estudio en 2008, comparan la influencia de expasyl, magic foam cordy Ultrapak (sin agente impregnado) sobre el te- jido gingival de 180 premolares de 60 pacientes, libre de signos de gingivitis. Observados después de la retracción, un día después y a los 7 días. A partir de esto determinaron que los tres sistemas causaron una lesión aguda después de un día, pero le tomo una semana a los grupos de magic foam cord y Ultrapak para la recuperación. La lesión fue significativamente mayor para expasyl y exhibió una recuperación más lenta al ser comparados los grupos, además advierte de la posibilidad de sensibilidad por su uso debido a un número pequeño de casos encontrados. Por último, se informa que Ultrapak no requirió de hemostático para el control del sangrado durante la retracción.

Para el 2010, Phatale et al (31), a través de su estudio, comparan el efecto de expasyl (pasta con 15\% cloruro de aluminio y caolín), magic foam cord (polivinil siloxano, inyectable) y un sistema de hilo retractor simple (Ultrapak) con $5 \%$ de cloruro de aluminio, sobre el epitelio del surco gingival de primeros premolares, en ambos maxilares, de 30 pacientes seleccionados para extracción con fines de ortodoncia. Se determinó que mientras para expasyl y magic foam cord sólo se observaron lesiones leves del epitelio, el hilo retractor causo lesiones moderadas en un $20 \%$.

El último grupo de técnicas de retracción revisadas son las contempladas en el área de la cirugía. El curetaje gingival rotatorio o gingitaje se considera como una técnica quirúrgica simple, donde por medio de instrumentos rotatorios diamantados operados a alta velocidad se puede otorgar una conformación a la pared interna del surco al tiempo que se prepara la terminación subgingival del diente $(9,11$, $12,16)$. Pero también se advierte de la posibilidad de daño al tejido o sobre extensión durante este método en comparación con otros $(9,27)$.

Con relación a esto, Kamansky et al (32), en 1984, en un estudio, compararon la respuesta del tejido gingival ante el gingitaje y un hilo retractor con Hemodent, en 10 pacientes adultos seleccionados para prótesis fija, con tres mediciones del surco vestibular y palatino: previo al procedimiento, a los 14 y 90 días después de este. Determinaron que el curetaje gingival rotatorio mostró significativamente menos cambios en la altura del margen gingival vesti- 
bular que el otro método. Por el contrario, en la superficie palatina no se observaron diferencias significativas. Además informan que el gingitaje supuso una aparente perturbación de la unión apical del epitelio del surco, con su posterior reparación y estabilización. Esto posiblemente por la falta de sensación táctil durante la instrumentación. Sin embargo, los cambios en la profundidad del surco no se registraron como clínicamente significativos. Adicionalmente, en un estudio anterior, Tupac y Neacy (33) en 1981 a través de una comparación entre el gingitaje y un sistema de hilo retractor en caninos de 15 perros adultos, evaluados en periodos de 0,7 y 21 días, no reportaron diferencias significativas entre estos métodos.

La siguiente técnica descrita es la electrocirugía, donde se utiliza el calor generado por una corriente eléctrica alterna de alta frecuencia a través de los tejidos, con múltiples propósitos como cauterización, hemostasia, destrucción y división (34). Este método provee espacio para la línea de terminación por eliminación no por retracción, donde un electrodo es guiado a través del tejido, sin la necesidad de presión. Cuando un refinamiento en el sitio de la cirugía es necesario se recomienda esperar por lo menos 5 segundos, para dispersión del calor, antes de aplicar nuevamente el electrodo $(16,35)$. Se informa de la capacidad de un buen control de la zona de coagulación durante el procedimiento (36), al actuar por desecación para cumplir con la hemostasia, donde el material celular es descompuesto en oxígeno, carbono, hidrógeno y nitrógeno dejando clínicamente un coágulo de color negro o carbonizado (35).

Existe un desacuerdo en opiniones sobre la posibilidad de daño al tejido y efectividad de este método al ser comparado con métodos mecánico-químicos, como hilos impregnados con epinefrina y no impregnados, donde se informa desde no existir diferencias significativas durante la comparación (37), hasta el reporte de perdida de altura del margen gingival y elevación de la presión sanguínea asociada al uso de electrocirugía (38).

En relación a lo anterior, Azzi et al (39) en 1983, en un estudio clínico e histológico donde compararon a la electrocirugía, el gingitaje y un sistema de hilo retractor no impregnado en 20 perros adultos, sobre sus tres incisivos maxilares y tomando el cuarto como control, valorados en intervalos de 6 horas, 1, 3, 7 y 14 días. Determinaron que todos los métodos ocasionaron algún tipo de daño en el epitelio del surco, pero solo una recesión de magnitud clínica y temporal fue observada con el curetaje gingival rotatorio. Aunque todos los cambios destructivos observados en los periodos iniciales se repararon al cabo de 14 días, por lo que la migración apical del margen no se observó para los métodos evaluados.

Por su parte, DeVitre et al (40), en 1985, a través de un estudio comparativo entre la electrocirugía y el gingitaje, en 20 pacientes adultos que necesitaban coronas completas superiores en el sector anterior, con evaluación del margen gingival en intervalos de 4, 8 y 12 semanas, concluyeron que la electrocirugía mostró mayor pérdida de tejido que la retracción con fresa, sin embargo el método de electrocirugía influyó en un mayor volumen del material de impresión en la zona del margen con relación al método comparado.

Otra posibilidad estudiada es la cirugía láser, a través de equipos con múltiples longitudes de onda y potencia como el láser de dióxido de carbono $\left(\mathrm{CO}_{2}\right)$, láser de diodos, láser Er:YAG y Nd:YAG, usados con múltiples propósitos en la odontología (41-44). En el manejo de tejidos en prótesis fija se emplea con el fin de eliminar el exceso de tejido del margen gingival de una futura restauración, al tiempo que se asegura la hemostasia y previene la contaminación por bacterias del área quirúrgica. Aunque por la posibilidad de interacción directa de la energía láser con la estructura dental a través del margen gingival libre se recomienda baja intensidad, y en algunos casos se puede considerar finalizar la separación con otro instrumento como una cureta o cucharilla afilada. La posibilidad de daños al tejido por falta de sensación táctil también es un factor a considerar (45). La literatura reporta al láser de diodo de $980 \mathrm{~nm}$ como un sistema con precisión en la incisión, con un efecto de separación comparable a los obtenidos con un láser de $\mathrm{CO}_{2}$ y una buena coagulación, similar al láser Nd:YAG $(46,47)$. Aun las zonas de daño térmico horizontal y vertical de estos sistemas se informan en un rango comparable (47). Adicionalmente se informa que el láser de $\mathrm{CO}_{2}$ en comparación con el láser de diodo, fomenta una mayor absorción de agua en los tejidos blandos, lo que podría reducir el 
efecto térmico de los tejidos adyacentes (48). Existe el reporte de un mayor daño lateral térmico del láser de dióxido de carbono en comparación con métodos como la electrocirugía (49).

Al confrontar la muestra $(21,23,30-32,41)$ en el contexto de la literatura consultada se puede inferir que los resultados no son homogéneos por lo que surgen distintas posiciones frente al uso de las técnicas disponibles. Basados en la evidencia se puede concluir que todos los métodos causan en algún grado lesiones al tejido gingival, algunos en mayor grado que otros y la capacidad de recuperación del tejido gingival varía de 7 a 14 días. La elección de un método depende del conocimiento integral de cada caso clínico en particular y no de procesos estandarizados basados en la superioridad de un método ante otro.

\section{BIBLIOGRAFÍA}

1. Shetty MS, Shenoy KK. Techniques for evaluating the fit of removable and fixed prosthesis. ISRN Dent 2011;2011:348372. Epub 2011;Jul 14.

2. Finger WJ, Kurokawa R, Takahashi H, Komatsu $M$. Sulcus reproduction with elastomeric impression materials: a new in vitro testing method. Dent Mater 2008;Dec;24(12):1655-60. Epub 2008;May 21.

3. Fazekas A, Csempesz F, Csabai Z, Vág J. Effects of pre-soaked retraction cords on the microcirculation of the human gingival margin. Oper Dent 2002;Jul-Aug;27(4):343-8.

4. Porzier J, Benner-Jordan L, Bourdeau B, Losfeld $R$. [Access to the intracrevicular space in preparations for fixed prosthesis]. Cah Prothese 1991;Mar;(73):6-20.

5. Dimashkieh MR, Morgano SM. A procedure for making fixed prosthodontic impressions with the use of preformed crown shells. J Prosthet Dent 1995;Jan;73(1):95-6.

6. Bowles WH, Tardy SJ, Vahadi A. Evaluation of new gingival retraction agents. J Dent Res 1991; Nov,70(11):1447-9.
7. Nowakowska D, Saczko J, Kulbacka J, Choromanska A. Dynamic oxidoreductive potential of astringent retraction agents. Folia Biol (Praha) 2010;56(6): 263-8.

8. Sábio S, Franciscone PA, Mondelli J. Effect of conventional and experimental gingival retraction solutions on the tensile strength and inhibition of polymerization of four types of impression materials. J Appl Oral Sci 2008;Jul-Aug;16(4):280-5.

9. Shannon A. Expanded clinical uses of a novel tissue-retraction material. Compend Contin Educ Dent 2002;Jan,23(1 Suppl):3-6; quiz 18.

10. Brady WF. Periodontal and restorative considerations in rotary gingival curettage. J Am Dent Assoc 1982;Aug;105 (2): 231-6.

11. Donovan TE, Chee WW. Current concepts in gingival displacement. Dent Clin North Am 2004; Apr;48(2):vi, 433-44.

12. Bennani V, Schwass D, Chandler N. Gingival retraction techniques for implants versus teeth: current status. J Am Dent Assoc. 2008;Oct;139 (10):1354-63.

13. Livaditis GJ. The matrix impression system for fixed prosthodontics. J Prosthet Dent. 1998;Feb; 79 (2):208-16.

14. Ferrari M, Cagidiaco MC, Ercoli C. Tissue management with a new gingival retraction material: a preliminary clinical report. J Prosthet Dent 1996;Mar;75(3):242-7.

15. Livaditis GJ. Comparison of the new matrix system with traditional fixed prosthodontic impression procedures. J Prosthet Dent.1998;Feb;79(2):200-7.

16. Benson BW, Bomberg TJ, Hatch RA, Hoffman W Jr. Tissue displacement methods in fixed prosthodontics. J Prosthet Dent 1986;Feb;55(2): 175-81.

17. Kumbuloglu O, User A, Toksavul S, Boyacioglu $\mathrm{H}$. Clinical evaluation of different gingival retraction cords. Quintessence Int. 2007;Feb;38(2):e92-8. 
18. Runyan DA, Reddy TG Jr, Shimoda LM. Fluid absorbency of retraction cords after soaking in aluminum chloride solution. J Prosthet Dent 1988; Dec;60(6) 676-8.

19. Cloyd S, Puri S. Using the double-cord packing technique of tissue retraction for making crown impressions. Dent Today 1999;Jan;18(1):54-9.

20. Csempesz F, Vág J, Fazekas A. In vitro kinetic study of absorbency of retraction cords. J Prosthet Dent 2003;Jan;89(1):45-9.

21. Weir DJ, Williams BH. Clinical effectiveness of mechanical-chemical tissue displacement methods. J Prosthet Dent 1984;Mar;51(3): 326-9.

22. Donovan TE, Gandara BK, Nemetz H. Review and survey of medicaments used with gingival retraction cords. J Prosthet Dent 1985;Apr;53(4): 525-31.

23. Csillag M, Nyiri G, Vag J, Fazekas A. Dose-related effects of epinephrine on human gingival blood flow and crevicular fluid production used as a soaking solution for chemo-mechanical tissue retraction. J Prosthet Dent 2007;Jan;97(1): 611.

24. Nemetz H, Donovan T, Landesman H. Exposing the gingival margin: a systematic approach for the control of hemorrhage. J Prosthet Dent 1984; May;51(5):647-51.

25. Goldberg AT, Yoder JL, Thayer KE. Analysis of heart rate in dogs during retraction and impression procedures. J Dent Res 1971;May-Jun;50(3):6458.

26. Jokstad A. Clinical trial of gingival retraction cords. J Prosthet Dent 1999;Mar;81(3):258-61.

27. Wassell RW, Barker D, Walls AW. Crowns and other extra-coronal restorations: impression materials and technique. Br Dent J 2002;Jun 29;192(12):679-84, 687-90.

28. Kopac I, Sterle M, Marion L. Electron microscopic analysis of the effects of chemical retraction agents on cultured rat keratinocytes. J Prosthet Dent 2002 Jan;87 (1): 51-6.

29. Chang YS, Bennani V, Tawse-Smith A, Girvan L. Effect of a cordless retraction paste material on implant surfaces: an in vitro study. Braz Oral Res 2011;Dec;25 (6): 492-9.

30. Al Hamad KQ, Azar WZ, Alwaeli HA, Said KN. A clinical study on the effects of cordless and conventional retraction techniques on the gingival and periodontal health. J Clin Periodontol 2008; Dec;35(12):1053-8.

31. Phatale S, Marawar PP, Byakod G, Lagdive SB, Kalburge JV. Effect of retraction materials on gingival health: A histopathological study. J Indian Soc Periodontol 2010;Jan;14(1):35-9.

32. Kamansky FW, Tempel TR, Post AC. Gingival tissue response to rotary curettage. J Prosthet Dent 1984;Sep;52(3):380-3.

33. Tupac RG, Neacy K. A comparison of cord gingival displacement with the gingitage technique. J Prosthet Dent 1981;Nov;46(5):509-15.

34. Sinha UK, Gallagher LA. Effects of steel scalpel, ultrasonic scalpel, $\mathrm{CO}_{2}$ laser, and monopolar and bipolar electrosurgery on wound healing in guinea pig oral mucosa. Laryngoscope 2003;Feb; 113(2):228-36.

35. Bashetty K, Nadig G, Kapoor S. Electrosurgery in aesthetic and restorative dentistry: A literature review and case reports. J Conserv Dent. 2009; Oct;12(4):139-44.

36. Flocken JE. Electrosurgical management of soft tissues and restorative dentistry. Dent Clin North Am. 1980;Apr;24(2):247-69.

37. Wöstmann B, Rehmann P, Trost D, Balkenhol M. Effect of different retraction and impression techniques on the marginal fit of crowns. J Dent 2008;Jul;36(7):508-12. Epub 2008 May 13.

38. Stark MM, Nicholson DJ, Soelberg KB, Kempler $\mathrm{D}$, Pelzner RB. The effects of retraction cords 
and electrosurgery upon blood pressure and tissue regeneration in rhesus monkeys. J Dent Res 1977;Aug;56(8):881-8.

39. Azzi R, Tsao TF, Carranza FA Jr, Kenney EB. Comparative study of gingival retraction methods. J Prosthet Dent 1983;Oct;50 (4):561-5.

40. DeVitre R, Galburt RB, Maness WJ. Biometric comparison of bur and electrosurgical retraction methods. J Prosthet Dent 1985 Feb;53(2):17982.

41. S ims s ek Kaya G, YapýcýYavuz G, Sümbüllü MA, Dayý E. A comparison of diode laser and Er:YAG lasers in the treatment of gingival melanin pigmentation. Oral Surg Oral Med Oral Pathol Oral Radiol Endod 2011;Jun 9. [Epub ahead of print]

42. Ozbayrak S, Dumlu A, Ercalik-Yalcinkaya S. Treatment of melanin-pigmented gingiva and oral mucosa by $\mathrm{CO}_{2}$ laser. Oral Surg Oral Med Oral Pathol Oral Radiol Endod 2000;Jul;90 (1):14-5.

43. Spitz SD. Lasers in prosthodontics: clinical realities of a dental laser in a prosthodontic practice. Alpha Omegan 2008;Dec;101(4):188-94.

44. Abdel Gabbar F, Aboulazm SF. Comparative study on gingival retraction using mechanochemical procedure and pulsed Nd = YAG laser irradiation. Egypt Dent J 1995;Jan;41(1):1001-6.

45. Parker S. The use of lasers in fixed prosthodontics. Dent Clin North Am 2004;Oct;48(4):971-98, viiviii.
46. Romanos G, Nentwig GH. Diode laser (980 nm) in oral and maxillofacial surgical procedures: clinical observations based on clinical applications. J Clin Laser Med Surg 1999;Oct;17(5):193-7.

47. Goharkhay K, Moritz A, Wilder-Smith P, Schoop U, Kluger W, Jakolitsch S, Sperr W. Effects on oral soft tissue produced by a diode laser in vitro. Lasers Surg Med 1999;25(5):401-6.

48. Cercadillo-Ibarguren I, España-Tost A, ArnabatDomínguez J, Valmaseda-Castellón E, BeriniAytés L, Gay-Escoda C. Histologic evaluation of thermal damage produced on soft tissues by $\mathrm{CO}_{2}$, Er,Cr:YSGG and diode lasers. Med Oral Patol Oral Cir Bucal 2010;Nov 1;15(6):e912-8.

49. Schoinohoriti OK, Chrysomali E, Iatrou I, Perrea D. Evaluation of lateral thermal damage and reepithelialization of incisional wounds created by $\mathrm{CO}(2)$-laser, monopolar electrosurgery, and radiosurgery: a pilot study on porcine oral mucosa. Oral Surg Oral Med Oral Pathol Oral Radiol Endod 2011 Sep 7. [Epub ahead of print]

\section{CORRESPONDENCIA}

Alberto Carlos Cruz González

Facultad de Odontología

Universidad de Cartagena

Campus de la Salud

Zaragocilla. Cartagena

Correos electrónicos: alcruzgo@gmail.com, adiazc1@unicartagena.edu.co 